This item was submitted to Loughborough's Research Repository by the author.

Items in Figshare are protected by copyright, with all rights reserved, unless otherwise indicated.

\title{
Fock-Darwin-like quantum dot states formed by charged Mn interstitial ions
}

PLEASE CITE THE PUBLISHED VERSION

http://dx.doi.org/10.1103/PhysRevLett.101.226807

\section{PUBLISHER}

(C) American Physical Society

VERSION

VoR (Version of Record)

LICENCE

CC BY-NC-ND 4.0

\section{REPOSITORY RECORD}

Makarovsky, O., O. Thomas, Alexander G. Balanov, Laurence Eaves, A. Patane, Richard Campion, Charles T. Foxon, et al.. 2019. "Fock-darwin-like Quantum Dot States Formed by Charged Mn Interstitial lons". figshare. https://hdl.handle.net/2134/12738. 
This item was submitted to Loughborough's Institutional Repository (https://dspace.lboro.ac.uk/) by the author and is made available under the following Creative Commons Licence conditions.

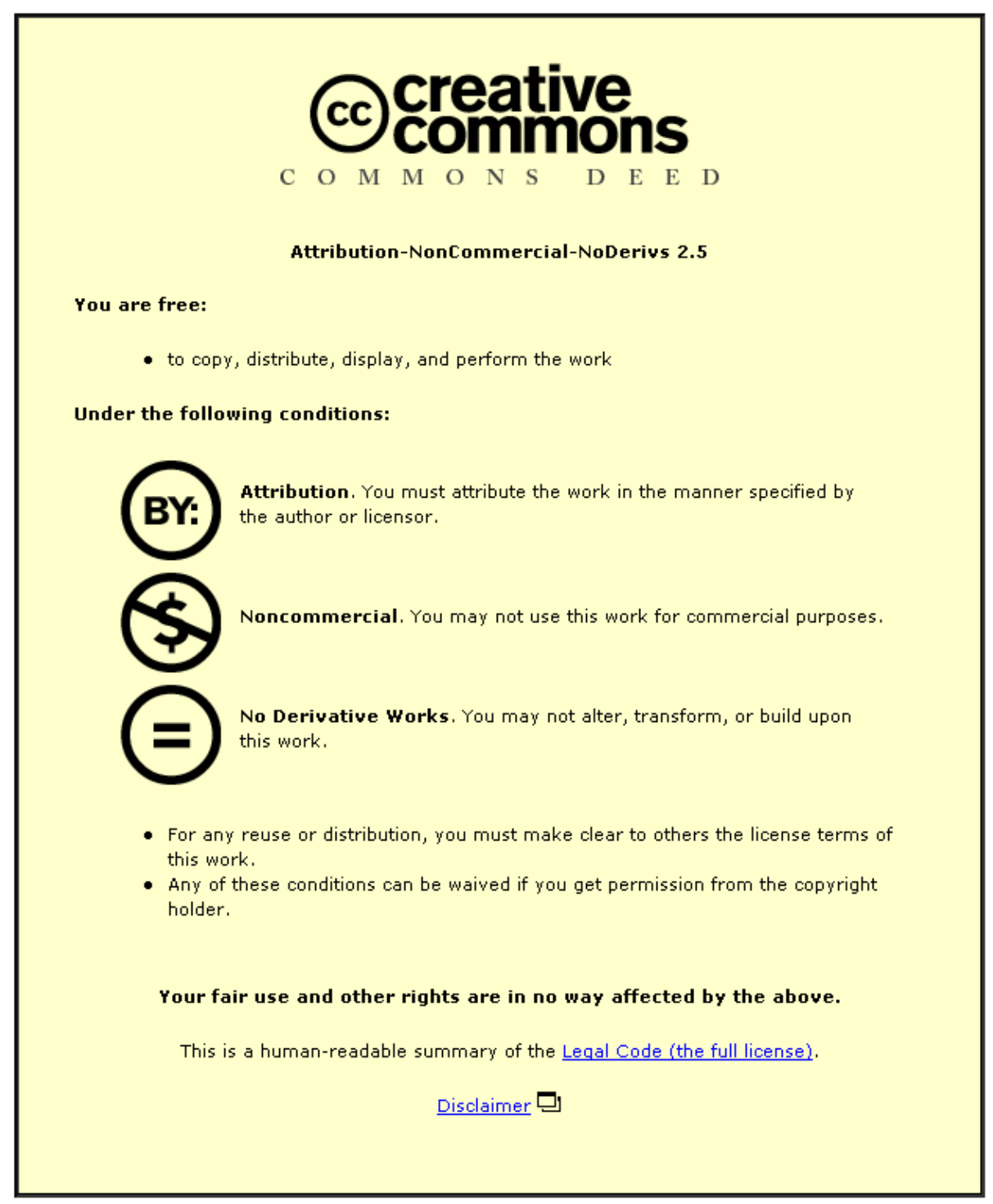

For the full text of this licence, please go to: http://creativecommons.org/licenses/by-nc-nd/2.5/ 


\title{
Fock-Darwin-Like Quantum Dot States Formed by Charged Mn Interstitial Ions
}

\author{
O. Makarovsky, ${ }^{1, *}$ O. Thomas, ${ }^{1}$ A. G. Balanov, ${ }^{1,2}$ L. Eaves,${ }^{1}$ A. Patanè, ${ }^{1}$ R. P. Campion, ${ }^{1}$ C. T. Foxon, ${ }^{1}$ E. E. Vdovin,,${ }^{1,3}$ \\ D. K. Maude, ${ }^{4}$ G. Kiesslich, ${ }^{1, \dagger}$ and R. J. Airey ${ }^{5}$ \\ ${ }^{1}$ School of Physics \& Astronomy, University of Nottingham, Nottingham NG7 2RD, United Kingdom \\ ${ }^{2}$ Department of Physics, Loughborough University, Loughborough LE11 3TU, United Kingdom \\ ${ }^{3}$ Institute of Microelectronic Technology RAS, Chernogolovka, 142432, Russia \\ ${ }^{4}$ Grenoble High Magnetic Field Laboratory, CNRS, F-38042 Grenoble, France \\ ${ }^{5}$ Department of Electronic \& Electrical Engineering, University of Sheffield, Sheffield S1 3JD, United Kingdom
}

(Received 8 July 2008; published 25 November 2008)

\begin{abstract}
We report a method of creating electrostatically induced quantum dots by thermal diffusion of interstitial Mn ions out of a p-type (GaMn)As layer into the vicinity of a GaAs quantum well. This approach creates deep, approximately circular, and strongly confined dotlike potential minima in a large $(200 \mu \mathrm{m})$ mesa diode structure without need for advanced lithography or electrostatic gating. Magnetotunneling spectroscopy of an individual dot reveals the symmetry of its electronic eigenfunctions and a rich energy level spectrum of Fock-Darwin-like states with an orbital angular momentum component $\left|l_{z}\right|$ from 0 to 11 .
\end{abstract}

DOI: 10.1103/PhysRevLett.101.226807

Quantum dots (QDs) are nanostructures in which the electronic motion is confined in all three spatial directions. This leads to quantization of the eigenstates into discrete energy levels [1]. Improved synthesis of new materials and nanoscale control of their physical properties have led to innovative approaches to the fabrication of QDs and their functionalization for specific applications. These methods include atom diffusion and implantation [2,3], nanolithography [4], electrostatic gating [5,6], self-assembly [7], and colloidal synthesis [8]. This Letter reports an alternative method of QD fabrication, which exploits the controlled thermal diffusion of $\mathrm{Mn}$ interstitial ions $\left(\mathrm{Mn}_{i}{ }^{2+}\right)$ out of a layer of ferromagnetic $(\mathrm{GaMn}) \mathrm{As}[9,10]$ into the underlying layer of a heterostructure containing a AlAs/GaAs/AlAs quantum well (QW). For a narrow range of annealing conditions, the random clustering of $\mathrm{Mn}_{i}{ }^{2+}$ ions that diffuse into the GaAs spacer layer between the (GaMn)As and QW modifies the electrostatic potential within the well, giving rise to a small number of deep potential energy minima which quantum-confine electrons on length scales, $l_{0} \sim 10 \mathrm{~nm}$. The magnetic field dependence of the tunnel current through individual dots reveals a rich spectrum and provides spatial images of the ground and excited state electron wave functions of the dot. By varying the annealing conditions and the separation between the $(\mathrm{GaMn})$ As layer and QW, it should be possible to prepare a dot with a specified degree of quantum confinement.

In (GaMn)As, Mn interstitials form when the Mn concentration exceeds $\sim 2 \%$. They are doubly ionised donors, $\mathrm{Mn}_{i}{ }^{2+}\left(3 d^{5}\right)$, and inhibit ferromagnetism as they compensate the itinerant holes introduced by substitutional $\mathrm{Mn}_{\mathrm{Ga}}$ acceptors; see Fig. 1(a) [11]. Thermal annealing of (GaMn) As is an effective means of increasing its Curie temperature and electrical conductivity by out-diffusion of $\mathrm{Mn}_{i}$ to the
PACS numbers: 73.22.-f, 73.63.Kv, 75.50.Pp, 75.75.+a

surface [12]. Here we exploit controlled annealing to create QD-like potential minima in $p-i-n$ resonant tunneling diodes (RTDs) with different Mn concentrations, grown by molecular beam epitaxy on (001) $n^{+}$-GaAs substrates [13] We focus on a structure with the following composition, in order of growth: a $300 \mathrm{~nm}$ layer of $n^{+}$-GaAs Si-doped to $2 \times 10^{18} \mathrm{~cm}^{-3} ; 100 \mathrm{~nm}$ of $n$-GaAs doped to $2 \times$ $10^{17} \mathrm{~cm}^{-3}$; an undoped central intrinsic region comprising a $20 \mathrm{~nm}$ GaAs layer, a $5 \mathrm{~nm}$ AlAs tunnel barrier, a $6 \mathrm{~nm}$

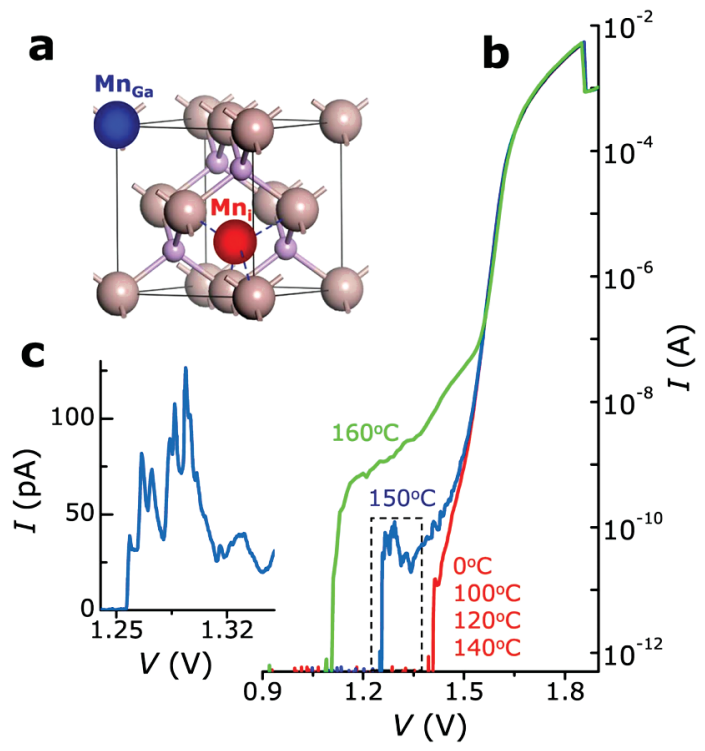

FIG. 1 (color). (a) Schematic showing the locations in the GaAs lattice of $\mathrm{Mn}_{\mathrm{Ga}}$ and $\mathrm{Mn}_{i}$ ions. (b) $I(V)$ plots at $T=4 \mathrm{~K}$ before annealing and following 3 -h anneals at temperatures up to $140{ }^{\circ} \mathrm{C}$ (red) and subsequent 3 -h anneals at $150{ }^{\circ} \mathrm{C}$ (blue) and $160{ }^{\circ} \mathrm{C}$ (green). (c) Detail showing the sharp peaks in $I(V)$ following the $150{ }^{\circ} \mathrm{C}$ anneal. 
GaAs QW, a $5 \mathrm{~nm}$ AlAs tunnel barrier and a $10 \mathrm{~nm} \mathrm{GaAs}$ layer; and, finally, a $50 \mathrm{~nm}$ capping layer of $p^{+}-(\mathrm{GaMn}) \mathrm{As}$ with $3 \% \mathrm{Mn}$. The $(\mathrm{GaMn}) \mathrm{As}$ layer was grown at $250{ }^{\circ} \mathrm{C}$, the others at $600^{\circ} \mathrm{C}$. The epilayers were processed into $200 \mu \mathrm{m}$ diameter mesa diodes.

Figure 1(b) shows the effect of progressive annealing on the forward bias current-voltage characteristics, $I(V)$, for one of our mesas (sample 1). Prior to annealing, the current increases rapidly at the flat band condition, $V \approx V_{\mathrm{FB}}=$ $1.41 \mathrm{~V}$ [13], rising to a peak at $1.8 \mathrm{~V}$, which corresponds to resonant electron tunneling through the lowest $(E 1)$ subband of the two-dimensional (2D) conduction band QW. No current is detectable above the background noise level $(<0.3 \mathrm{pA})$ in the as-grown mesas for $V<1.3 \mathrm{~V}$. However, following annealing at $150{ }^{\circ} \mathrm{C}$ for 3 hours, sharp peaks appear in $I(V)$; see Fig. 1(c). We have investigated similar peaks in the $I(V)$ curves of a large number of other annealed samples. The thermal broadening of the low voltage threshold of each peak between 0.3 and $20 \mathrm{~K}$ confirms that they arise from resonant tunneling from the Fermi energy, $E_{F}$, of a thermalized degenerate electron gas of the $n$-GaAs emitter into a single quantized energy level [14]. These measurements also determine the fraction of applied voltage, $f=0.65 \pm 0.05$, dropped between $E_{F}$ and the level. This $f$-value indicates that the states through which electrons tunnel are spatially confined between the two AlAs barriers; the energy of a particular state is given by efV.

Higher temperature (up to $200{ }^{\circ} \mathrm{C}$ ) or longer annealing ( $>3$ hours) leads to merging of the sharp peaks into an increasingly broad and strong shoulder in $I(V)$; see Fig. 1(b). We attribute this to the formation of additional current pathways arising from diffusion of increasing numbers of $\mathrm{Mn}_{i}$ ions from the (GaMn)As towards the QW. This conclusion is supported by measurements on control samples, in which a Be- or C-doped $p$-GaAs capping layer replaces the $(\mathrm{GaMn}) \mathrm{As}$ : their $I(V)$ are unaffected by annealing for $24 \mathrm{~h}$ at $250{ }^{\circ} \mathrm{C}$.

To understand the nature of the quantum states associated with the sharp tunneling resonances, we examine the effect of a magnetic field, $B_{z}$, applied perpendicular to the QW plane. Figure 2(a) shows a gray-scale plot of differential conductance, $d I / d V$, versus $B_{z}$ for a sample (sample 2) annealed at $150{ }^{\circ} \mathrm{C}$ for $3 \mathrm{~h}$; alongside is the $I(V)$ curve at $B_{z}=28 \mathrm{~T}$; see Fig. 2(b). The lowest-energy peak does not split, but shifts to higher bias, consistent with the diamagnetic shift of a $1 s$-like orbital ground state. In contrast, the higher bias peaks reveal a rich orbital Zeeman splitting pattern. Figure 2(a) also plots the calculated $B_{z}$ dependence of the Fock-Darwin (FD) spectrum for a 2D parabolic potential [15]. This provides a quantitative fit to our data with only one adjustable parameter, $\hbar \omega_{0}=$ $22 \pm 2 \mathrm{meV}$, the energy spacing of the FD levels at $B_{z}=$ 0 . The other parameters are the electron effective mass in GaAs and leverage factor $f$.

The emergence, at high field $\left(B_{z}>16 \mathrm{~T}\right)$, of a series of equally-spaced peaks, which shift to lower energy and

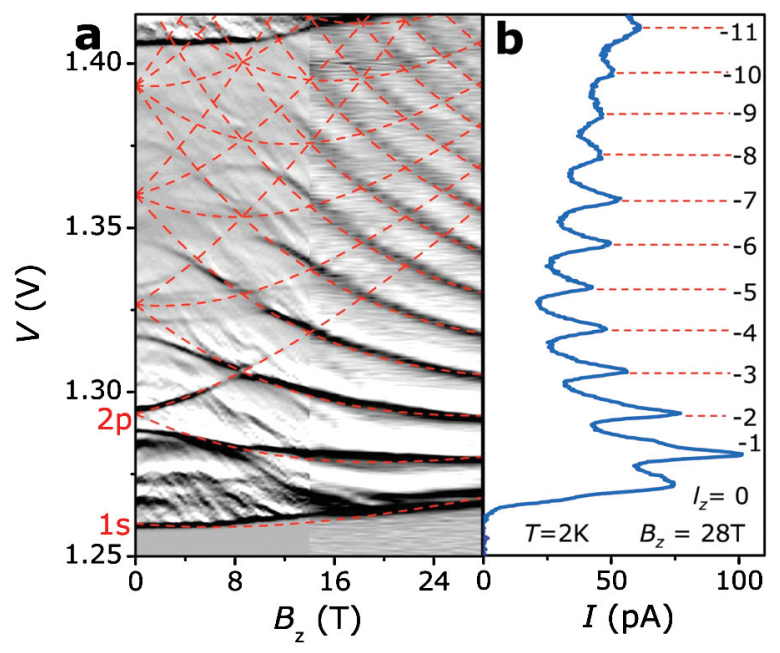

FIG. 2 (color). (a) Gray-scale plot of $d I / d V$ at $T=2 \mathrm{~K}$ versus $B_{z}$ up to $28 \mathrm{~T}$. Data above $B_{z}>14 \mathrm{~T}$ are taken using a resistive magnet, leading to higher electrical noise and a slight decrease of resolution above $14 \mathrm{~T}$. (b) $I(V)$ curve at $B_{z}=28 \mathrm{~T}$.

become more closely spaced with increasing $B_{z}$, is a characteristic feature of FD states. At high $B_{z}$, these evolve into nondegenerate states of the lowest Landau level, which skip around equipotential contours of the confining potential with different quantized angular momenta, $l_{z}$; we observe $\left|l_{z}\right|$ values from 0 to 11 [Fig. 2(b)]. A comparison of our data with the FD model reveals that the confining potential does not have exact circular symmetry: the orbital degeneracy of the first two excited states, $2 p_{ \pm}$, is lifted even at $B_{z}=0$ (see the split peaks at $V \sim 1.29 \mathrm{~V}$ ). This lowered symmetry also explains the lifting of the threefold orbital degeneracy of the next set of excited states around $V=1.325 \mathrm{~V}$ and the level anticrossings, e.g., that at $V=$ $1.31 \mathrm{~V}$ and $B_{z}=8 \mathrm{~T}$.

From our measurements, we deduce that the electrons are tunneling through the bound states of a deep and approximately parabolic circular potential well. To understand how such a potential can be produced by low temperature annealing, we use Fick's law to calculate the density distribution of $\mathrm{Mn}_{i}$ ions that have diffused out of the $p-(\mathrm{GaMn}) \mathrm{As}$, assuming a constant surface concentration of $\mathrm{Mn}_{i}$ at the $(\mathrm{GaMn}) \mathrm{As} / \mathrm{GaAs}$ interface. This gives the mean concentration, $C(z)=C_{0} \operatorname{erfc}\left(z / 2 \sqrt{D t_{A}}\right)$, of $\mathrm{Mn}_{i}$ at a distance $z$ below the interface. Here $C_{0}=5 \times$ $10^{19} \mathrm{~cm}^{-3}$ is the concentration of $\mathrm{Mn}_{i}$ in the $(\mathrm{GaMn}) \mathrm{As}$ layer for $3 \% \mathrm{Mn}$ [11], $D=D_{0} \exp \left(-Q / k_{B} T_{A}\right)$ is the $\mathrm{Mn}_{i}$ diffusion coefficient, $D_{0}=3 \times 10^{-4} \mathrm{~m}^{2} / \mathrm{s}, Q=1.5 \mathrm{eV}$ [12], and $T_{A}$ and $t_{A}$ are the annealing temperature and time, respectively. The band bending resulting from the diffused $\mathrm{Mn}_{i}{ }^{2+}$ ions is calculated from Poisson's equation and shown in Fig. 3(a). Although the conduction band edge $E_{c}$ in the GaAs spacer layer is lowered relative to the unannealed case, it remains well above the Fermi energy $E_{F}$, so that the $\mathrm{Mn}_{i}{ }^{2+}$ donors remain ionized. Despite the 

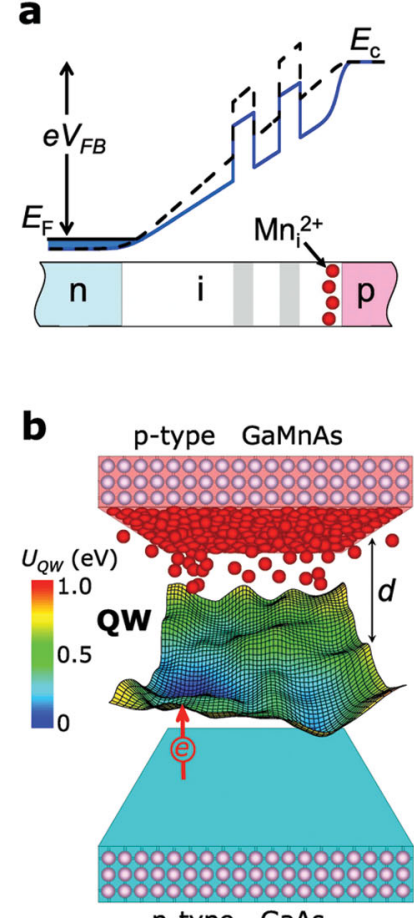

n-type GaAs
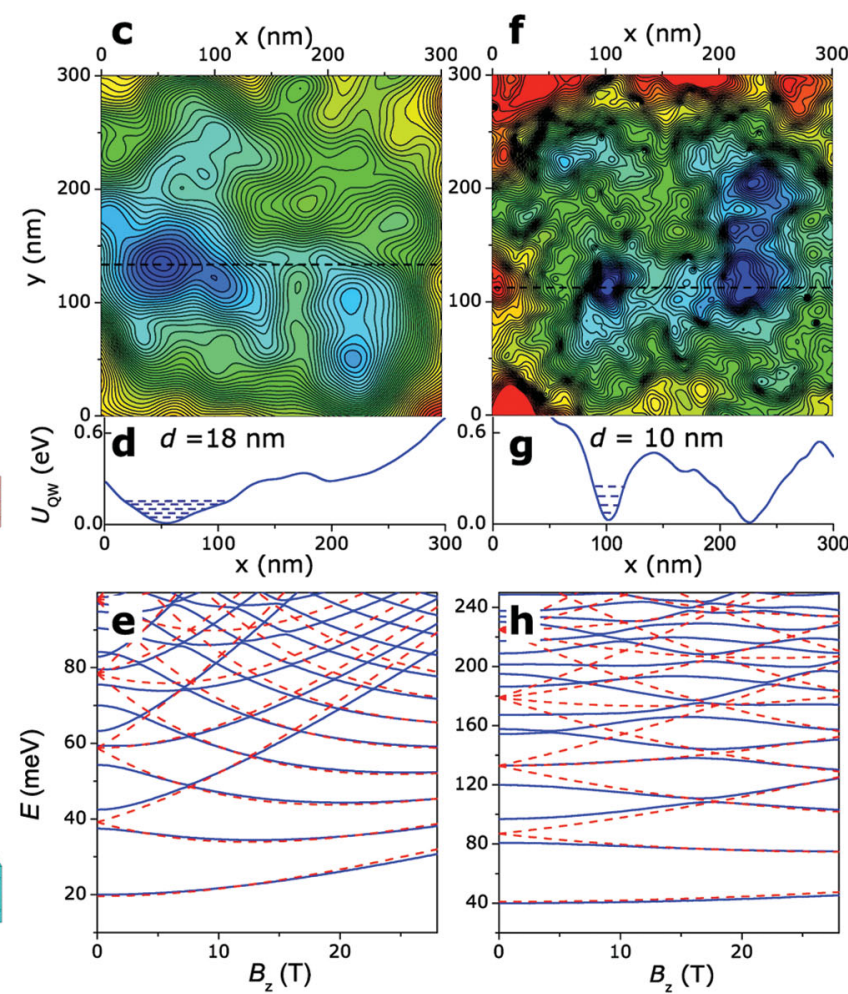

FIG. 3 (color). (a) Conduction band diagram of our device before (black) and after (blue) annealing. (b) Potential energy profile, $U_{\mathrm{QW}}(x, y)$, in the central QW plane, and schematic of an electron tunneling from the $n$-GaAs layer into quantized states of a minimum of $U_{\mathrm{QW}}(x, y)$. (c), (f) Numerical simulations of $U_{\mathrm{QW}}(x, y)$ for $d=18$ and $10 \mathrm{~nm}$. (d),(g) Variation of $U_{\mathrm{QW}}(x, y)$ along the dashed lines in plots (c) and (f); dashed blue lines indicate the lowest-energy levels of one of the minima. (e),(h) Blue solid curves: calculated $B_{z}$ dependences of the energies of the electronic bound states shown in plots (d) and (g). Red dashed lines: FD spectra. electric field in the intrinsic region of the diode, the barrier height, $Q$, for hopping between interstitial sites is only slightly reduced $(<5 \%)$ by electrostatic effects. This is supported by experiment: we observe no change in the diffusion rate of $\mathrm{Mn}_{i}$ for annealing at $150{ }^{\circ} \mathrm{C}$ in the presence of applied voltages of $\pm 1 \mathrm{~V}$.

We simulate numerically the distribution of $\mathrm{Mn}_{i}$ ions neglecting Coulomb interactions between them. This is a reasonable approximation since the $\mathrm{Mn}_{i}$ density in the GaAs spacer is relatively low, changing from $\sim 10^{19} \mathrm{~cm}^{-3}$ at the (GaMn)As interface to effectively zero at the AlAs interface (corresponding to a mean $\mathrm{Mn}_{i}$ separation $>5 \mathrm{~nm}$ and a Coulomb interaction energy much less than $Q$ ). Our simulated positions of individual $\mathrm{Mn}_{i}$ in the $x y$-plane are defined by random numbers corresponding to "white noise" of uniform distribution, with the ion density along the growth axis $z$ given by $C(z)$. The simulations use $3 \times 10^{6} \mathrm{Mn}_{i}{ }^{2+}$ ions occupying a volume of $5 \times 5 \times d \mu \mathrm{m}^{3}$, where $d$ is the distance from the (GaMn)As/GaAs interface to the central QW plane. Figures 3(b) and 3(c) show the resulting electronic potential energy $U_{\mathrm{QW}}(x, y)$ in the middle of the $\mathrm{QW}$, for a central area of $0.3 \times 0.3 \mu \mathrm{m}^{2}$, over which edge effects can be neglected. The potential profile and energy contours in Figs. 3(b) and 3(c) reveal a complex landscape. This arises from random spatial variations in the density profile of diffused $\mathrm{Mn}_{i}$ with nanoscale regions ("clusters") extending over $\sim 10 \mathrm{~nm}$, where the local density of $\mathrm{Mn}_{i}$ considerably exceeds the mean value. The clusters giving rise to the deepest potential minima typically contain up to $\sim 10$ randomly-placed $\mathrm{Mn}_{i}$ ions and form in the GaAs layer just below the $(\mathrm{GaMn})$ As layer. A deep minimum in $U_{\mathrm{QW}}(x, y)$ directly below such a cluster can be seen to left of center in Fig. 3(c) and in the section along $x$ through the minimum, Fig. 3(d). The lowest-energy quantized states of this potential minimum are $\sim 0.3 \mathrm{eV}$ below $E_{c}$. Hence they provide resonant channels for electron tunneling at bias voltages far below the flat band condition and give rise to the sharp peaks in $I(V)$. By solving numerically the 2D Schrödinger equation of an electron in this minimum as a function of $B_{z}$, we obtain the magnetospectrum in Fig. 3(e). This is in good qualitative agreement with the measured spectrum in Fig. 2(a).

A comparison of the simulations in Figs. 3(c), 3(d), 3(f), and $3(\mathrm{~g})$ shows how it is possible to adjust the typical depth and curvature of the minima in $U_{\mathrm{QW}}(x, y)$ : reducing $d$ from 18 to $10 \mathrm{~nm}$ increases the $\hbar \omega_{0}$ of a typical deep potential minimum from $\sim 20$ to $\sim 45 \mathrm{meV}$ [see Figs. 3(e) and 3(h)], in qualitative agreement with an analysis based on Poisson's equation.

The spatial forms of the QD wave functions are related directly to the potential landscape by Schrödinger's equation. By measuring the tunnel current through a particular QD as a function of $\boldsymbol{B}_{x y}$ aligned along different directions in the $x y$ plane, we obtain a spatial map of the probability density $|\psi(\boldsymbol{k})|^{2}$ of an eigenstate in momentum space [14]. The Lorentz force on the tunneling electrons allows us to tune the in-plane momentum, $\boldsymbol{k}$, according to the relation $\boldsymbol{k}=-e \boldsymbol{s} \times \boldsymbol{B} / \hbar$, where $s=10 \pm 1 \mathrm{~nm}$ is the length of the tunneling path from the emitter into the bound states of the QD. Figure 4(a) shows spatial images of the ground and first two excited states of the QD potential obtained using 
a
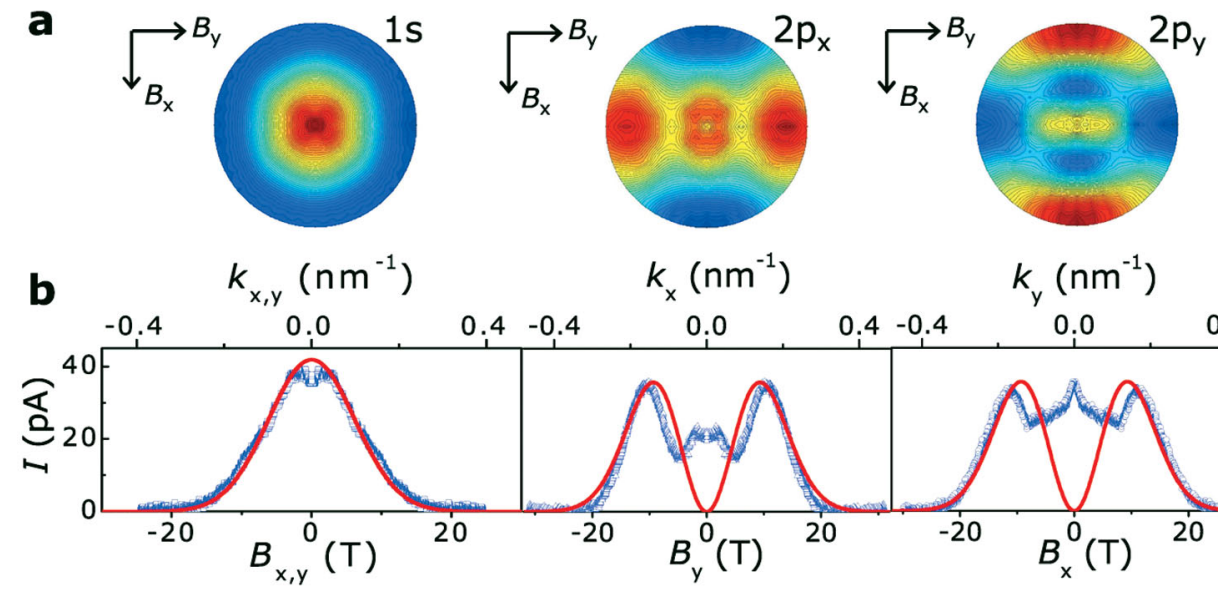

$k_{y}\left(\mathrm{~nm}^{-1}\right)$

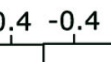

0.0 0.4 fields up to $14 \mathrm{~T}$, and $|k|$ up to $0.2 \mathrm{~nm}^{-1}$. Whereas the ground state wave function has approximately circular symmetry, the wave function maps of the first two excited states have the lobed character of $2 p_{x}$ and $2 p_{y}$ orbitals rather than the toroidal shape of degenerate $2 p_{ \pm}$orbitals. This is consistent with the observed zero field energy splitting of the $2 p$-like states in Fig. 2(a) and provides additional evidence that the symmetry of the confining potential is lower than that of the FD model.

Figure 4(b) probes the $|\psi(\boldsymbol{k})|^{2}$ of the $2 p_{x, y}$ orbitals along their symmetry axes to higher $k$ values using fields up to $30 \mathrm{~T}$. A fit to the simple harmonic oscillator (SHO) approximation gives $\hbar \omega_{0}=24 \pm 2 \mathrm{meV}$, in agreement with the value $22 \mathrm{meV}$ obtained independently from Fig. 2(a). However, the measured $|\psi(\boldsymbol{k})|^{2}$ of the $2 p_{x, y}$ states also have a significant amplitude at $\boldsymbol{k}_{x y} \sim \boldsymbol{B}_{x y}=0$, in contrast to the form expected for single particle SHO states. We tentatively attribute this feature to electron-electron interactions between the tunneling electron and nearby electrons in the emitter Fermi sea [16-18]. Finally, we note the presence in our differential conductance plots of additional peaks, which have a different $B$ dependence from the FDlike resonances, e.g., between the $1 s$ and $2 p$ peaks in Figs. 1(c) and 2(a). Their $B$ dependence in both field configurations indicates that they are related to electron quantization effects of the weakly disordered $n$-GaAs emitter states [19].

In summary, we have shown how, in a (GaMn)As $p-i-n$ RTD, the controlled diffusion of ionized Mn interstitials leads to the formation of well-defined GaAs quantum dots with a deep confining potential. We observe a rich spectrum of Fock-Darwin-like states, with orbital angular momenta $\left|l_{z}\right|$ up to 11 . By adjusting the annealing conditions and/or the separation between the $p$-(GaMn)As layer and the GaAs QW, it should be possible to control the depth of the QD potential and the characteristic spacing, $\hbar \omega_{0}$, of its electronic energy levels. Our approach could be exploited to form low-dimensional confinement potentials in func- tional spintronic devices containing (GaMn)As or other materials, and to study fundamental science, e.g., imaging the wave functions of QDs containing more than one electron $[20,21]$.

We acknowledge helpful discussions with our colleagues K. W. Edmonds, B. L. Gallagher, and T. Jungwirth, and the support of the EPSRC (UK), the Royal Society, and RFBR (Russia).

*Corresponding author.

oleg.makarovsky@nottingham.ac.uk

†Present address: Institut für Theoretische Physik, Technische Universität Berlin, D-10623 Berlin, Germany.

[1] D. Bimberg, M. Grundmann, and N. N. Ledentsov, Quantum Dot Heterostructures (Wiley, New York, 1999).

[2] F. E. Prins et al., Appl. Phys. Lett. 63, 1402 (1993).

[3] L. Besombes et al., Phys. Rev. Lett. 93, 207403 (2004).

[4] M. A. Reed et al., Phys. Rev. Lett. 60, 535 (1988).

[5] R. C. Ashoori et al., Phys. Rev. Lett. 71, 613 (1993).

[6] S. Tarucha et al., Phys. Rev. Lett. 77, 3613 (1996).

[7] L. Goldstein et al., Appl. Phys. Lett. 47, 1099 (1985).

[8] A. P. Alivisatos, Science 271, 933 (1996).

[9] T. Dietl et al., Science 287, 1019 (2000).

[10] Y. Ohno et al., Nature (London) 402, 790 (1999).

[11] T. Jungwirth et al., Phys. Rev. B 72, 165204 (2005).

[12] K. W. Edmonds et al., Phys. Rev. Lett. 92, 037201 (2004).

[13] For a description of the optical and electrical properties of our devices prior to annealing, see O. Thomas et al., Appl. Phys. Lett. 90, 082106 (2007).

[14] A. Patanè et al., Phys. Rev. B 65, 165308 (2002).

[15] B. L. Johnson and G. Kirczenow, Europhys. Lett. 51, 367 (2000).

[16] A. K. Geim et al., Phys. Rev. Lett. 72, 2061 (1994).

[17] I. Hapke-Wurst et al., Phys. Rev. B 62, 12621 (2000).

[18] E. E. Vdovin et al., Phys. Rev. B 75, 115315 (2007).

[19] B. Jouault et al., Phys. Rev. B 73, 155415 (2006).

[20] M. Rontani and E. Molinari, Phys. Rev. B 71, 233106 (2005).

[21] G. Maruccio et al., Nano Lett. 7, 2701 (2007). 